\title{
ERRATUM
}

\section{Erratum to: Movement of carabids from grassy strips to crop land in organic agriculture}

\author{
Mazhar Hussain Ranjha • Ulrich Irmler
}

Published online: 22 August 2014

(c) Springer International Publishing Switzerland 2014

\section{Erratum to: J Insect Conserv (2014) 18:457-467}

DOI 10.1007/s10841-014-9657-1

The original article was published with typesetting errors in

Table 1. The corrected table is given in this erratum.

Table 1 Total number of carabids released in the fields, total recaptured in different crops $(\mathrm{W}=$ wheat, $\mathrm{C}=$ clover, $\mathrm{P}=$ pumpkin $)$ and in adjacent strips ( $\mathrm{S}=$ grassy strip, and $\mathrm{H}=$ strip with hedge); T.rec.: total of recaptured

\begin{tabular}{|c|c|c|c|c|c|c|c|c|c|c|c|c|c|c|c|}
\hline \multirow[t]{4}{*}{ Species } & \multicolumn{5}{|l|}{ Field 1} & \multicolumn{5}{|l|}{ Field 2} & \multicolumn{4}{|l|}{ Field 3} & \multirow{4}{*}{$\begin{array}{l}\text { Total } \\
\text { strips } \\
(\%)\end{array}$} \\
\hline & Released & Reca & ture & & T. rec. & Released & $\operatorname{Rec}$ & aptur & & T. rec. $(\%)$ & Released & Recaptured & & T. rec. & \\
\hline & \multicolumn{4}{|c|}{ Year 2010 (autumn) } & & & & & & & & & & & \\
\hline & & $\mathrm{W}$ & & $\mathrm{S}$ & & & $\mathrm{C}$ & $\mathrm{P}$ & $S$ & & & & & & \\
\hline P. melanarius & 488 & 147 & & 26 & 36 & 382 & 83 & 15 & 18 & 30 & & & & & \\
\hline$P$. niger & 55 & 8 & & 14 & 40 & 60 & 2 & 1 & 4 & 12 & & & & & \\
\hline \multirow[t]{3}{*}{ N. brevicollis } & 44 & 1 & & 0 & 2 & 105 & 13 & 5 & 0 & 17 & & & & & \\
\hline & \multicolumn{4}{|c|}{ Year 2011 (spring) } & & & & & & & & & & & \\
\hline & & $\mathrm{C}$ & $\mathrm{H}$ & $S$ & & & & & & & & $\mathrm{~W}$ & $S$ & & \\
\hline P. melanarius & 2,046 & 141 & 39 & 44 & 11 & & & & & & 1,826 & 179 & 75 & 14 & 33 \\
\hline C. auratus & 53 & 21 & 6 & 3 & 57 & & & & & & 190 & 85 & 20 & 55 & 21 \\
\hline C. nemoralis & 45 & 3 & 8 & 1 & 27 & & & & & & 5 & 0 & 0 & 0 & 75 \\
\hline Poecilus spp. & 236 & 34 & 3 & 18 & 24 & & & & & & 763 & 63 & 12 & 12 & 34 \\
\hline
\end{tabular}

The online version of the original article can be found under doi:10. 1007/s10841-014-9657-1.

M. H. Ranjha ( $₫)$ · U. Irmler

University of Kiel, Kiel, Schleswig-Holstein, Germany

e-mail: mranjha@ecology.uni-kiel.de 